\title{
Familial Nonmedullary Thyroid Carcinoma (Fnmtc): Molecular Advances with the New Sequencing Technologies
}

\author{
Erika Urbano de Lima ${ }^{1,2 *}$, Mariana Teixeira Rodrigues ${ }^{1,2}$, Ileana Gabriela Sanchez de Rubió ${ }^{1,2,3}$ \\ ${ }^{1}$ Thyroid Molecular Science Laboratory, Universidade Federal de São Paulo, Brazil \\ ${ }^{2}$ Universidade Federal de São Paulo, Brazil
}

${ }^{3}$ Biological Sciences Department, Universidade Federal de São Paulo, Brazil

Submission: February 03, 2017; Published: June 21, 2018

*Corresponding author: Erika Urbano de Lima, Thyroid Molecular Science Laboratory, Programa de pós-graduação em Biologia Estrutural e Funcional, Universidade Federal de São Paulo, Brazil, Email: erika.urbanodelima@gmail.com

\section{Introduction}

Approximately 3-9\% of the thyroid neoplasms are hereditary. Familial non-medullary thyroid cancer (FNMTC) is diagnosed when three or more first-degree relatives are affected and is classified into Syndromic familial adenomatous polyposis, Gardner's syndrome, Cowden's disease, Carney's complex type 1, Werner's syndrome) and Nonsyndromic (thyroid cancer). Several candidate chromosomal loci and susceptibility genes have been reported but these results are not replicated in subsequent studies. As the Next Generation Sequencing (NGS) now offers a powerful new diagnostic approach of the goal of this article was to review the current knowledge the new approaches used for the molecular characterization of FNMTC cases.

Familial predisposition of nonmedullary thyroid carcinoma (FNMTC) is diagnosed when three or more first-degree relatives are affected. It occurs in about 3-9\% of the follicular cell-derived neoplasms and is classified into Syndromic and Nonsyndromic because encompasses a heterogeneous group of diseases [1]. The Syndromic group is characterized by a predominance of non-thyroidal tumors. Some syndromes are more frequently observed, such as familial adenomatous polyposis (FAP), Cowden syndrome, Werner syndrome and Carney complex, with thyroid cancer prevalence of $2-12 \%, 35 \%, 18 \%$ and $15 \%$, respectively. Except for Werner's syndrome, all syndromes related with FNMTC are autosomal dominant. Several driver-genes have been identified, APC gene was associated to FAP and PTEN, SDH, PIK3CA, AKT1 and KLLN were related to Cowden syndrome; PRKAR1 $\alpha$ was related to Carney complex and WRN gene was associated to Werner's syndrome [1].

The Nonsyndromic form accounts for 95\% of all FNMTC cases and is characterized by the presence of thyroid cancer and the absence of other known associated syndromes [2]. The Papillary Thyroid Carcinoma (PTC) subtype is the most commonly observed and may or may not be associated with benign thyroid neoplasms (multinodular goiter) or autoimmunity thyroid disease (Hashimoto's thyroiditis). Several candidate chromosomal loci and susceptibility genes have been reported (1q21, 2q21, 6q22, 8p23.1-p22 and 19p13.2, NKX2-1, FOXE1, SRGAP1, TERT, HABP2 and C14orf93) suggesting that it is a polygenic familial cancer syndrome [1,3-5]. As subsequent studies failed to identify segregation of these candidates with the disease and due to the clinical variability of the patients, the molecular profile of each FNMTC family may be unique [6-9]. Furthermore, important cancer-related genes (APC, PTEN, TSHR, RET, TRK, c-MET, BRAF and H-K-N-RAS) were also excluded as the cause of FNMTC [10]. In the last decade, techniques for single-target detection have been replaced by the Next Generation Sequencing (NGS), which allows simultaneous analysis of a large group of genes generating a large volume of data in parallel [11-13]. The NGS high-throughput platforms are more efficient, less expensive and provides information that is not provided by Sanger DNA sequencing analysis or by hot spot mutation gene targeted assays (MLPA - Multiplex Ligation Probedependent Amplification or Taqman Genotyping) [10-12]. Analysis of the generated data is a complex process. In familial diseases with clinical heterogeneity, as observed in FNMCT, a careful selection of the individuals to be submitted to the NGS is necessary. The inclusion of unaffected individuals improves diagnostic rates by facilitating variants selection and mutations identification due to the exclusion of regional polymorphisms. The definition of a suitable pipeline for the identification and classification of the variants is also an essential step $[11,12]$. In silico predictive analysis provided by specific programs such as Polyphen2, SIFT, Mutation Assessor and Mutation Taster is a valuable tool to distinguish between the pathological and benign variants associated with the phenotype, taking into account the evolutionary conservation of the amino acid or nucleotide residue, 
biochemical impact of the amino acid substitution considering the physicochemical properties, and the variant localization $[11,12]$. As there is no rule, the use a more stringent filtering criterion are more appropriate as the first approach while less strict selection may be adequate when no candidate variant was identified [13-16]. It is noteworthy that a consensus among the prediction results leads to a better accuracy, as in CONDEL, PON-P and Meta-SNP algorithms $[11,17,18]$. Information obtained from population databases (1000 Genomes Project, ExAc and Exome Variant Server (EVS)) are frequently used to exclude variants that are deemed polymorphic/benign based on a global minor allele frequency (MAF) cut off of $\sim 1 \%(0.01)$.

However, it is important to consider ethnicity-specific MAFs that depend on the ethnic background of the population, particularly in populations with high miscegenation as observed in Brazilians, underrepresented in most of the genomic databases, however data made available by local consortiums as ABraOM [19] and EPIGEN-BRAZIL [20] unravel this problem. In the literature, it is already possible to observe the benefits of new generation sequencing in the FNMTC. Recently, a group sought to identify previously undescribed cancer-predisposing gene(s) in a Cowden Syndrome family enriched for thyroid cancer across 4 generations, who had tested negative for PTEN, SDHB-D and KLLN, via an approach combining exome sequencing and family study. They found a variant in SEC23B gene and suggested that this germline heterozygous variant is associate with cancer predisposition [21]. Moreover, when whole-genome approach was conducted in sporadic FAP patient in which any pathogenic APC mutations was found by the conventional Sanger sequencing, a mosaic mutation in $\sim 12 \%$ of his peripheral leukocytes was identified. Demonstrating that NGS is an effective tool to identify genetic mosaicism in hereditary diseases [22]. A variant on WRN gene was identified by exome-wide sequencing in a 16-year-old girl with an atypical syndrome without diagnosis after 10 years of the first symptoms. The variant has been previously reported in Werner's syndrome (WS) and when the patient was re-evaluated several features of WS were detected, allowing the early diagnosis of a recessive disease and the early use of adequate therapies and interventions [23]. Using Whole Exome Sequencing (WES) a germline variant in the HABP2 gene was identified in a family with seven PTC patients of a FNMTC kindred and in $4.7 \%$ of 423 sporadic PTC cases. This variant increased protein expression in the tumor samples and functional studies showed that the loss of function p.G534E rs7080536 variant leads to increased colony formation and cell migration, characteristics of malignant transformation, suggesting that the HABP2 gene may be involved in susceptibility to thyroid cancer [4]. Because the authors did not report the ethnicity of the family and for having used only one database to determine MAF (1000 Genomes), this result was questioned by others groups [24]. Furthermore, this variant was considered a polymorphism in other populations (United Kingdom, USA, Saudi Arabia, Colombia, Spain, Italy, Australia and Brazil) [24].
Only Zhang et al identified the rs7080536 variant in 4/29 (13.8\%) of unrelated FNMTC kindreds [25]. In other study, through linkage analysis and exome-sequencing the genes C14orf93 (RTFC), PYGL and BMP4 were identified as candidate genes in a FNMTC family with 5 cases of PTC. However, the functional studies showed that only the p.V205M mutation of the C14orf93 gene led to increased migration rate and colony formation in a PTC cell line [5]. Next Generation Sequencing allied to capture of expressed sequences from genomic DNA now offers a powerful new diagnostic approach. Barriers to use this technology still include cost and the complexity of interpreting results arising from simultaneous identification of large numbers of variants. Thus, cost reductions and new friendly analysis of this big data are necessary. Even so, the results obtained through this new generation sequencing technology have opened doors to new possibilities in the search for the molecular bases of thyroid familial cancer.

\section{References}

1. Guilmette J, Nosé V (2018) Hereditary and familial thyroid tumours. Histopathology 72(1): 70-81.

2. Nosé V (2011) Familial thyroid cancer: a review. Mod Pathol 24(Suppl 2): S19-S33.

3. Pereira JS, da Silva JG, Tomaz RA, Pinto AE, Bugalho MJ, et al. (2015) Identification of a novel germline FOXE1 variant in patients with familial non-medullary thyroid carcinoma (FNMTC). Endocrine 49(1): 204-214.

4. Gara SK, Jia L, Merino MJ, Agarwal SK, Zhang L, et al. (2015) Germline HABP2 Mutation Causing Familial Nonmedullary Thyroid Cancer. N Engl J Med 373: 448-455.

5. Liu C, Yu Y, Yin G, Zhang J, Wen W, et al. (2017) C14orf93 ( RTFC) is identified as a novel susceptibility gene for familial nonmedullary thyroid cancer. Biochem Biophys Res Commun 482(4): 590-596.

6. de Mello LEB, Araujo AN, Alves CX, de Paiva FJP, Brandão-Neto J, et al. (2017) The G534E variant in HABP 2 is not associated with increased risk of familial nonmedullary thyroid cancer in Brazilian Kindreds. Clin Endocrinol (Oxf) 87(1): 113-114.

7. Colombo C, Muzza M, Proverbio MC, Ercoli G, Perrino M, et al. (2017) Segregation and expression analyses of hyaluronan-binding protein 2 (HABP2): insights from a large series of familial non-medullary thyroid cancers and literature review. Clin Endocrinol (Oxf) 86(6): 837844.

8. Weeks AL, Wilson SG, Ward L, Goldblatt J, Hui J, et al. (2016) HABP2 germline variants are uncommon in familial nonmedullary thyroid cancer. BMC Med Genet 17(1): 60.

9. Marques IJ, Moura MM, Cabrera R, Pinto AE, Simões-Pereira J, et al (2017) Identification of somatic TERT promoter mutations in familial nonmedullary thyroid carcinomas. Clin Endocrinol (Oxf) 87(4): 394399.

10. Roca I, Fernández-Marmiesse A, Gouveia S, Segovia M, Couce M (2018) Prioritization of Variants Detected by Next Generation Sequencing According to the Mutation Tolerance and Mutational Architecture of the Corresponding Genes. Int J Mol Sci 19(6): 1584.

11. Li MM, Datto M, Duncavage EJ, Kulkarni S, Lindeman NI, et al. (2017) Standards and Guidelines for the Interpretation and Reporting of Sequence Variants in Cancer: A Joint Consensus Recommendation of the Association for Molecular Pathology, American Society of Clinical Oncology, and College of American Pathologists. J Mol Diagn 19(1): 4-23. 
12. Bahassi EM, Stambrook PJ (2014) Next-generation sequencing technologies: breaking the sound barrier of human genetics. Mutagenesis 29(5): 303-310.

13. Tranchevent LC, Capdevila FB, Nitsch D, De Moor B, De Causmaecker P, et al. (2011) A guide to web tools to prioritize candidate genes. Brief Bioinform 12(1): 22-32.

14. Jalali Sefid Dashti M, Gamieldien J (2017) A practical guide to filtering and prioritizing genetic variants. Biotechniques 62(1): 18-30.

15. Flanagan SE, Patch AM, Ellard S (2010) Using SIFT and PolyPhen to Predict Loss-of-Function and Gain-of-Function Mutations. Genet Test Mol Biomarkers 14(4): 533-537.

16. Dong C, Wei P, Jian X, Gibbs R, Boerwinkle E, et al. (2015) Comparison and integration of deleteriousness prediction methods for nonsynonymous SNVs in whole exome sequencing studies. Hum Mol Genet 24(8): 2125-2137.

17. Capriotti E, Nehrt NL, Kann MG, Bromberg Y (2012) Bioinformatics for personal genome interpretation. Brief Bioinform 13(4): 495-512.

18. Capriotti E, Altman RB, Bromberg Y (2013) Collective judgment predicts disease-associated single nucleotide variants. BMC Genomics 14(Suppl 3): S2.

19. Naslavsky MS, Yamamoto GL, de Almeida TF, Ezquina SAM, Sunaga DY, et al. (2017) Exomic variants of an elderly cohort of Brazilians in the ABraOM database. Hum Mutat 38(7): 751-763.

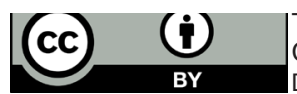

This work is licensed under Creative Commons Attribution 4.0 Licens DOI: 10.19080/JETR.2018.03.555618
20. Lima-Costa MF, Rodrigues LC, Barreto ML, Gouveia M, Horta BL, et al. (2015) Genomic ancestry and ethnoracial self-classification based on 5,871 community-dwelling Brazilians (The Epigen Initiative). Sci Rep 5: 9812 .

21. Yehia L, Niazi F, Ni Y, Ngeow J, Sankunny M, et al. (2015) Germline Heterozygous Variants in SEC23B Are Associated with Cowden Syndrome and Enriched in Apparently Sporadic Thyroid Cancer. Am J Hum Genet 97(5): 661-676.

22. Yamaguchi K, Komura M, Yamaguchi R, Imoto S, Shimizu E, et al. (2015) Detection of APC mosaicism by next-generation sequencing in an FAP patient. J Hum Genet 60(5): 227-231.

23. Raffan E, Hurst LA, Turki S Al, Carpenter G, Scott C, et al. (2011) Early Diagnosis of Werner's Syndrome Using Exome-Wide Sequencing in a Single, Atypical Patient. Front Endocrinol (Lausanne) 2: 1-8.

24. Gerhard GS, Bann DV, Broach J, Goldenberg D (2017) Pitfalls of exome sequencing: a case study of the attribution of HABP2 rs7080536 in familial non-medullary thyroid cancer. NPJ Genomic Med 2: 8.

25. Zhang T, Xing M (2016) HABP2 G534E Mutation in Familial Nonmedullary Thyroid Cancer. J Natl Cancer Inst 108(6): djv415. 